Pacific Journal of Mathematics

COMPARISON THEOREMS FOR DELAY DIFFERENTIAL We 


\title{
COMPARISON THEOREMS FOR DELAY DIFFERENTIAL EQUATIONS
}

\author{
W. E. MAHFOUD
}

Comparison Theorems. This paper presents a technique for extending some oscillation results from ordinary to delay differential equations. According to the technique, if an oscillation result is known for the ordinary differential equation

$$
x^{(n)}+f(t, x)=0,
$$

the corresponding result for the delay differential equation

$$
x^{(n)}(t)+f(t, x(q(t)))=0
$$

may be obtained by a simple change of variables.

The conditions on $q$ and $f$ are mild and in the case of a bounded delay it is shown that the above equations have the same oscillatory behavior.

1. Introduction. We consider the equation

$$
x^{(n)}(t)+f(t, x(q(t)))=0
$$

where $n \geqq 2$. We let $R^{+}=[0,+\infty)$ and $R=(-\infty,+\infty)$ and assume throughout this paper that $q$ and $f$ satisfy the following conditions. $q: R^{+} \rightarrow R$ and $f: R^{+} \times R \rightarrow R$ are continuous, $q(t) \leqq t$ for $t \geqq 0$, $q(t) \rightarrow \infty$ as $t \rightarrow \infty, f(t, x)$ in nondecreasing in $x$, and $x f(t, x)>0$ if $x \neq 0$.

We label the above conditions on $q$ and $f$ as Hypothesis (E) for a future reference.

For any $t_{0} \geqq 0$, we let $E_{t_{0}}=\left\{s \mid s=q(t) \leqq t_{0}\right.$ for some $\left.t \geqq t_{0}\right\} \cup\left\{t_{0}\right\}$. By a solution of (1) at $t_{0}$ is meant a function $x: E_{t_{0}} \cup\left[t_{0}, t_{1}\right) \rightarrow R$, $t_{1}>t_{0}$, which satisfies (1) for all $t \in\left[t_{0}, t_{1}\right)$. All solutions of (1) at $t_{0}$ are assumed to exist on $\left[t_{0}, \infty\right)$ for every $t_{0} \geqq 0$. As in the case of ordinary differential equations, the existence of solutions on (1) on $\left[t_{0}, \infty\right)$ are usually guaranteed by requiring some growth conditions on $f$. For details, see [3].

A solution $x(t)$ of (1) at $t_{0}$ is said to be oscillatory if $x(t)$ has zeros for arbitrarily large $t$ and nonoscillatory if there exists $t^{*} \geqq t_{0}$ such that $x(t) \neq 0$ for all $t \geqq t^{*}$. Equation (1) is said to be oscillatory if every solution of (1) is oscillatory.

Most of the oscillation results which have appeared in the literature for delay differential equations are generalizations of known results for ordinary differential equations. Very often the method 
of proof of a generalized result is the same as that of the original result, but sometimes requiring a severe restriction on the delay. We propose in this paper to solve such generalized problems by reducing the study of the oscillatory properties of solutions of Equation (1) to that of an ordinary differential equation so that desirable generalizations of some oscillation criteria from ordinary to delay equations of the same types become immediate. In $\S 3$ we give three illustrative applications of our technique by deriving some results in [7], [9], and [12] from the corresponding ones in ordinary differential equations. Consequently we improve the results in [9] by relaxing the upper bound restriction on $q(t)$ and show that for a bounded delay Equation (1) and the corresponding ordinary equation

$$
x^{(n)}(t)+f(t, x)=0
$$

have the same oscillatory behavior.

2. Main results. We need the following two lemmas; the first one is essentially Kiguradze's lemma [6]. For a proof, see [10].

LEMma 1. Suppose $x(t)$ is a solution of (1) which is of constant sign on $\left[t_{0}, \infty\right), t_{0} \geqq 0$. Then there exists $t_{1} \geqq t_{0}$ such that on $\left[t_{1}, \infty\right)$ we have and

(i ) $x^{(k)}(t) x(t)>0$ whenever $k+n$ is odd and $0 \leqq k \leqq n-1$,

(ii) there exists an integer $l, 0 \leqq l \leqq n-1, n+l$ is odd, such that $x^{(k)}(t) x(t)>0$ for $k=0,1, \cdots, l,(-1)^{n+k-1} x^{(k)}(t) x(t)>0$ for $k=$ $l+1, \cdots, n-1$, and $x^{(n)}(t) x(t) \leqq 0$.

Lemma 2. Suppose $q$ and $f$ satisfy Hypothesis (E). If the differential inequality

$$
z^{\prime}(t)-f(t, z(q(t))) \geqq 0
$$

has a positive solution on $[\alpha, \infty)$, for some $\alpha>0$, so does the equation

$$
z^{\prime}(t)-f(t, z(q(t)))=0
$$

Proof. Let $z(t)$ be a positive solution of (3) on $[\alpha, \infty)$. Choose $t_{1} \geqq \alpha$ so that $q(t) \geqq \alpha$ for $t \geqq t_{1}$. Then $z(t)$ satisfies the inequality

$$
z(t) \geqq z\left(t_{1}\right)+\int_{t_{1}}^{t} f(u, z(q(u))) d u .
$$




$$
\begin{aligned}
y_{1}(t) & =z(t) \text { for } t \geqq \alpha \text { and } \\
y_{n}(t) & =z(t) \text { for } t \in\left[\alpha, t_{1}\right] \\
& =z\left(t_{1}\right)+\int_{t_{1}}^{t} f\left(u, y_{n-1}(q(u))\right) d u \text { for } t \geqq t_{1}
\end{aligned}
$$

and $n=2,3, \cdots$.

It follows from the definition of $y_{n}$ and (5) that the sequence $\left\{y_{n}\right\}$ satisfies the property that $z(t)=y_{1}(t) \geqq y_{2}(t) \geqq \cdots \geqq z\left(t_{1}\right)$ for all $t \geqq t_{1}$. Hence $\left\{y_{n}\right\}$ converges pointwise to a function $y(t)$ where $z(t) \geqq$ $y(t) \geqq z\left(t_{1}\right)$ for all $t \geqq t_{1}$. Let $f_{n}(t)=f\left(t, y_{n}(q(t))\right), n=1,2, \cdots$. Then $f_{1}(t) \geqq f_{2}(t) \geqq \cdots \geqq 0$. Since $f_{1}$ is integrable on $\left[t_{1}, t\right]$ for any $t \geqq t_{1}$ and $\lim _{n \rightarrow \infty} f_{n}(u)=f(u, y(q(u)))$ for any $u \in\left[t_{1}, t\right]$, then, by the monotone convergence theorem, we have

$$
y(t)=z\left(t_{1}\right)+\int_{t_{1}}^{t} f(u, y(q(u))) d u \text { for } t \geqq t_{1} .
$$

Hence $y(t)$ satisfies (4) and the proof is complete.

In connection with the study of solutions of Equation (1), we consider solutions of the equation

$$
x^{(n)}(t)+f^{*}(t, x(q(t)))=0
$$

where $f^{*}$ is defined by

$$
\begin{aligned}
& f^{*}(t, x)=f(t, x) \quad \text { if } \quad x \leqq 0 \\
& =-f(t,-x) \text { if } x \geqq 0 .
\end{aligned}
$$

It is clear from the definition of $f^{*}$ that $f^{*}(t,-x)=-f^{*}(t, x)$ and that $x f^{*}(t, x)>0$ if $x \neq 0$. Also, if $x(t)$ is a solution of (6), so is $-x(t)$; furthermore, $y(t)<0$ is a solution of (6) if and only if $y(t)$ is a solution of (1).

THEOREM 1. Suppose $q$ is continuously differentiable on $[\alpha, \infty)$, $\alpha \geqq 0$, and $q^{\prime}(t)>0$.

If, for $n$ even, Equation (1) has a nonoscillatory solution, then the equation

$$
\frac{d^{n} y}{d s^{n}}+\frac{1}{q^{\prime}\left(q^{-1}(s)\right)} f\left(q^{-1}(s), y\right)=0
$$

has a nonoscillatory solution.

If, for $n$ odd, Equation (1) has an unbounded nonoscillatory solution, so does Equation (7).

Proof. Let $x(t)$ be a nonoscillatory solution of (1) and assume 
$x(t)>0$ for $t \geqq t_{0}, t_{0} \geqq 0$. By Lemma 1 , there exists $t_{1} \geqq t_{0}$ and an integer $l, 0 \leqq l \leqq n-1, n+l$ is odd, such that $x^{(k)}(t)>0, k=$ $0,1, \cdots, l, \quad(-1)^{n+k-1} x^{(k)}(t)>0, k=l+1, \cdots, n-1$, and $x^{(n)}(t) \leqq 0$ for $t \geqq t_{1}$. Thus, if $n$ is even, or $n$ is odd and $x(t)$ is unbounded, then $l \geqq 1$. Choose $t_{1} \geqq \alpha$ and $t_{2} \geqq t_{1}$ so that $q(t) \geqq t_{1}$ for $t \geqq t_{2}$ and integrate (1) from $s$ to $\tau, \tau \geqq s \geqq t_{2}$, to obtain

$$
x^{(n-1)}(\tau)-x^{(n-1)}(s)+\int_{s}^{\tau} f(u, x(q(u))) d u=0
$$

and hence

$$
x^{(n-1)}(s) \geqq \int_{s}^{\infty} f(u, x(q(u))) d u \text { for all } s \geqq t_{2} .
$$

Let $V=q(u)$; then the above inequality yields

$$
x^{(n-1)}(s) \geqq \int_{q(s)}^{\infty}\left[f\left(q^{-1}(v), x(v)\right) / q^{\prime}\left(q^{-1}(v)\right)\right] d v, \quad s \geqq t_{2} .
$$

Since $q(t) \leqq t$, then

$$
x^{(n-1)}(s) \geqq \int_{s}^{\infty}\left[f\left(q^{-1}(v), x(v)\right) / q^{\prime}\left(q^{-1}(v)\right)\right] d v, \quad s \geqq t_{2} .
$$

Define

$$
F_{1} x(t)=\int_{t}^{\infty}\left[f\left(q^{-1}(v), x(v)\right) / q^{\prime}\left(q^{-1}(v)\right)\right] d v
$$

And

$$
F_{i} x(t)=\int_{t}^{\infty} F_{i-1} x(s) d s, \quad i=2,3, \cdots, n-l .
$$

Then it follows from (8) that the operators $F_{i}, i=1, \cdots, n-l$ are well-defined and that $F_{i} x(t) \geqq F_{i} y(t), i=1, \cdots, n-l$, whenever $x(t) \geqq y(t)$ and $t \geqq t_{2}$. Furthermore, $F_{i} x(t)>0$ and

$$
\frac{d}{d t} F_{i} x(t)=-F_{i-1} x(t), \quad t \geqq t_{2} \quad \text { and } \quad i=2, \cdots, n-l .
$$

Thus (8) reduces to

$$
x^{(n-1)}(s) \geqq F_{1} x(s), \quad s \geqq t_{2} .
$$

By successive integrations of this inequality from $s$ to $\tau, \tau \geqq s \geqq t_{2}$, discarding positive terms, we obtain

$$
(-1)^{i+1} x^{(n-i)}(s) \geqq F_{i} x(s), \quad s \geqq t_{2} \text { and } i=1, \cdots, n-l .
$$

In particular,

$$
x^{(l)}(s) \geqq F_{n-l} x(s), \quad s \geqq t_{2} .
$$


If we let $T_{0} x(t)=F_{n-l} x(t)$ and

$$
T_{i} x(t)=\int_{t_{2}}^{t} T_{i-1} x(s) d s, \quad t \geqq t_{2} \text { and } i=1, \cdots, l,
$$

then $T_{i} x(t) \geqq T_{i} y(t), \quad i=1, \cdots, l$, whenever $x(t) \geqq y(t)$ and $t \geqq t_{2}$. Furthermore, $T_{i} x(t)>0$ and

$$
\frac{d}{d t} T_{i} x(t)=T_{i-1} x(t), \quad t \geqq t_{2} \text { and } i=1, \cdots, l .
$$

By successive integrations of (10) from $t_{2}$ to $s$, discarding positive constants, we obtain

$$
x^{(l-i)}(s) \geqq T_{i} x(s), \quad s \geqq t_{2} \text { and } i=0,1, \cdots, l-1 .
$$

In particular,

$$
x^{\prime}(s) \geqq T_{l-1} x(s), \quad s \geqq t_{2} .
$$

By Lemma 2, the equation $x^{\prime}(s)=T_{l-1} x(s)$ has a positive solution $y(s)$ on $\left[t_{2}, \infty\right)$ such that $y^{\prime}(s)>0$ for all $s \geqq t_{2}$. By successive differentiations of the equation $y^{\prime}(s)=T_{l-1} y(s)$ using (9) and (11), we obtain

$$
y^{(l)}(s)=T_{0} y(s)=F_{n-l} y(s)
$$

and hence

$$
y^{(n-1)}(s)=F_{1} y(s)=\int_{s}^{\infty}\left[f\left(q^{-1}(v), y(v)\right) / q^{\prime}\left(q^{-1}(v)\right)\right] d v .
$$

Thus $y(s)$ satisfies Equation (7). If $n$ is odd, then $y^{\prime \prime}(s)>0$ and hence $y(s)$ is unbounded.

Now, assume $x(t)<0$ for $t \geqq t_{0}$ and let $u(t)=-x(t)$; then $u(t)$ is a positive solution of Equation (6) which is unbounded if $n$ is odd and $x(t)$ is unbounded. By the conclusion above, the equation

$$
y^{(n)}+\left[1 / q^{\prime}\left(q^{-1}(s)\right)\right] f^{*}\left(q^{-1}(s), y\right)=0
$$

has a positive solution $v(s)$. Let $y(s)=-v(s)$; then $y(s)$ is a solution of (12) and hence of (7) and which is unbounded when $n$ is odd. The proof is now complete.

The following theorem is an extension of [9, Theorems 6 and 15] to Equation (1). The result we obtain is essentially a comparison result between Equation (1) and the delay equation

$$
x^{(n)}(t)+f(t, x(Q(t)))=0
$$

where $Q: R^{+} \rightarrow R$ is continuous, $Q(t) \leqq t$ for $t \geqq 0$, and $Q(t) \rightarrow \infty$ as $t \rightarrow \infty$. The purpose of this extension is, on one hand, to relax the 
conditions of smoothness and monotonicity on $q(t)$ and, on the other hand, to show that for a bounded delay Equation (1) and Equation (2) have the same oscillatory behavior.

Theorem 2. Suppose $q(t) \geqq Q(t), t \geqq 0$.

If, for $n$ even, Equation (1) has nonoscillatory solution, so does Equation (13).

If, for $n$ odd, Equation (1) has an unbounded nonoscillatory solution, so does Equation (13).

Proof. Let $x(t)$ be a nonoscillatory solution of (1) and assume $x(t)>0$ for $t \geqq t_{0}, t_{0} \geqq 0$. It follows as in the proof of Theorem 1 that there exists $t_{2} \geqq t_{0}$ such that

$$
x^{(n-1)}(s) \geqq \int_{s}^{\infty} f(u, x(q(u))) d u, \quad s \geqq t_{2} .
$$

Define the sequence of operators $F_{i}(x, q)$ and $T_{i}(x, q)$ respectively by

$$
\begin{aligned}
& F_{1}(x, q)(t)=\int_{t}^{\infty} f(u, x(q(u))) d u \\
& F_{i}(x, q)(t)=\int_{t}^{\infty} F_{i-1}(x, q)(u) d u, \quad i=2, \cdots, n-l,
\end{aligned}
$$

and

$$
\begin{aligned}
& T_{0}(x, q)(t)=F_{n-l}(x, q)(t) \\
& T_{i}(x, q)(t)=\int_{t_{2}}^{t} T_{i-1}(x, q)(u) d u, \quad i=1, \cdots, l .
\end{aligned}
$$

It is clear that these functions satisfy the differentiation properties of (9) and (11) as well as the monotonicity property in both arguments $x$ and $q$. Hence, by successive integrations as in the proof of Theorem 1, we obtain

$$
x^{\prime}(t) \geqq T_{l-1}(x, q)(t), \quad t \geqq t_{2} .
$$

Since $q(t) \geqq Q(t)$, then $x^{\prime}(t) \geqq T_{l-1}(x, Q)(t)$ and hence, by Lemma 2 , the equation

$$
x^{\prime}(t)=T_{l-1}(x, Q)(t)
$$

has a nonoscillatory solution $y(t)$ which is unbounded when $n$ is odd. By successive differentiations of the equation $y^{\prime}(t)=T_{l-1}(y, Q)(t)$, we conclude that $y(t)$ satisfies Equation (13).

Now, if we assume $x(t)<0$ for $t \geqq t_{0}$, then the equation

$$
x^{(n)}(t)+f^{*}(t, x(Q(t)))=0
$$


has a positive solution $v(t)$ which is unbounded when $n$ is odd and $x(t)$ is unbounded. Hence $y(t)=-v(t)$ is a solution of (14) which satisfies (13). The proof is now complete.

Results similar to Theorem 2 have also been obtained in [5] and [11].

THeOREM 3. Suppose $q(t) \geqq Q(t), Q$ is continuously differentiable, and $Q^{\prime}(t)>0$ for $t \geqq \alpha, \alpha \geqq 0$.

If, for $n$ even, the equation

$$
\frac{d^{n} y}{d s^{n}}+\frac{1}{Q^{\prime}\left(Q^{-1}(s)\right)} f\left(Q^{-1}(s), y\right)=0
$$

is oscillatory, so is Equation (1).

If, for $n$ odd, Equation (15) has no bounded nonoscillatory solutions, neither does Equation (1).

Proof. It follows from Theorems 1 and 2.

Corollary. Suppose $c t \leqq q(t) \leqq t$ for some $c \in(0,1]$.

If, for $n$ even, the equation

$$
x^{(n)}+c^{n-1} f(t, x)=0
$$

is oscillatory, so is Equation (1).

If, for $n$ odd, Equation (16) has no bounded nonoscillatory solutions, neither does Equation (1).

Proof. Take $Q(t)=c t$ and set $s=Q(t)$ and $y(s)=x(t)$ in (15). Then $d^{n} y / d s^{n}=1 / c^{n} d^{n} x / d t^{n}$ and hence (15) reduces to (16).

If we let $\tau(t)=t-q(t)$, then Equation (1) may be written as

$$
x^{(n)}(t)+f(t, x(t-\tau(t)))=0 .
$$

The following result is concerned with bounded delays.

THEOREM 4. Suppose $\tau(t)$ is bounded. Then, for $n$ even, Equation (17) is oscillatory if and only if Equation (2) is oscillatory. For $n$ odd, every nonoscillatory solution of (17) is bounded if and only if every nonoscillatory solution of (2) is bounded.

Proof. Suppose $\tau(t) \leqq M$ for some $M>0$. Let $Q(t)=t-M$, $s=Q(t)$, and $y(s)=x(t)$; then $t \geqq q(t) \geqq Q(t)$ and $d^{n} y / d s^{n}=d^{n} x / d t^{n}$. Hence, the result follows from Theorems 2 and 3. 
REMARK. For $n$ odd, the terminology "strongly decreasing" has been used in $[8,9]$ to describe a solution $x(t)$ of (1) which satisfies $(-1)^{k} x^{(k)}(t)>0, k=0,1, \cdots, n-1$. It is then obvious that a nonoscillatory solution $x(t)$ of (1) is strongly decreasing if and only if it is bounded.

Brands [2] obtained Theorem 4 for $n=2$.

\section{Applications.}

(a) We first consider the nonlinear delay equation

$$
x^{(n)}(t)+a(t) f(x(q(t)))=0
$$

where $n \geqq 2, n$ is even, $a$ is continuous with $a(t) \geqq 0, f$ is continuously differentiable with $f^{\prime}(x) \geqq 0$, and $x f(x)>0$ if $x \neq 0$.

Kamenev [4] gave the following oscillation criterion for Equation (18) when $q(t)=t$.

THEOREM A. Suppose there exists a nondecreasing continuously differentiable function $\phi:(0, \infty) \rightarrow(0, \infty)$ such that

(i ) $\int^{ \pm \infty}\left[\phi\left(|x|^{1 / n-1}\right) f(x)\right]^{-1} d x<\infty \quad$ and

(ii) $\int^{\infty}\left[t^{n-1} a(t) / \phi(t)\right] d t=\infty$.

Then the equation $x^{(n)}+a(t) f(x)=0$ is oscillatory.

This result has been generalized by Kusano and Onose [7] to Equation (18) for any $q(t) \leqq t$ with $q^{\prime}(t) \geqq 0$. We show below that their generalization follows from Theorem 3. Indeed, by Theorem 3, Equation (18) is oscillatory if the corresponding ordinary equation

$$
\frac{d^{n} x}{d s^{n}}+\left[a\left(q^{-1}(s)\right) / q^{\prime}\left(q^{-1}(s)\right)\right] f(x)=0
$$

is oscillatory. By Theorem A, Equation (19) is oscillatory if conditions

(i ) and

(ii $)^{\prime} \int^{\infty}\left[s^{n-1} a\left(q^{-1}(s)\right) /\left(q^{\prime}\left(q^{-1}(s)\right) \phi(s)\right)\right] d s=\infty$

are satisfied. If we now let $s=q(t)$, then (ii)' reduces to

$$
\text { (ii })^{\prime \prime} \quad \int^{\infty}\left[q^{n-1}(t) a(t) / \phi(q(t))\right] d t=\infty .
$$

Thus (i) and (ii)" imply that Equation (19) and hence Equation (18) are oscillatory. This is precisely the result obtained in [7]. However, Theorem 3 requires that $q^{\prime}(t)>0$ while the condition on $q(t)$ in [7] is $q^{\prime}(t) \geqq 0$. This difference is insignificant especially when oscillation is described by divergent integrals such as the one in 
(ii)". We show below that our result which is obtained for $q^{\prime}(t)>0$ extends easily to the case $q^{\prime}(t) \geqq 0$. To see this, we assume that $q^{\prime}(t) \geqq 0$ and that (i) and (ii)" are satisfied. We let $q_{1}(t)=$ $\left(1-e^{-t}\right)^{1 / n-1} q(t)$ and consider the equation

$$
x^{(n)}(t)+a(t) f\left(x\left(q_{1}(t)\right)\right)=0 .
$$

It is clear that $q_{1}(t) \leqq q(t), q_{1}(t) \rightarrow \infty$ as $t \rightarrow \infty$, and $q_{1}^{\prime}(t)>0$. Hence, by Theorem 2, oscillation of Equation (18) follows from oscillation of Equation (20). Since $q_{1}^{\prime}(t)>0$, then, by the above result, Equation (20) is oscillatory if (i) and

(ii $)^{\prime \prime \prime} \int^{\infty}\left[q_{1}^{n-1}(t) a(t) / \phi\left(q_{1}(t)\right)\right] d t=\infty$

are satisfied. It remains only to show that (ii)" implies (ii)"'. Since $\phi$ is nondecreasing, then $\phi\left(q_{1}(t)\right) \leqq \phi(q(t))$. From the definition of $q_{1}(t)$ we have eventually $q_{1}^{n-1}(t) \geqq q^{n-1}(t) / 2$ and hence $q_{1}^{n-1}(t) a(t) / \phi\left(q_{1}(t)\right) \geqq$ $q^{n-1}(t) a(t) /[2 \phi(q(t))]$. Thus (ii)" implies (ii)"' and the proof is complete.

(b) We now consider the linear delay equation

$$
x^{(n)}(t)+a(t) x(q(t))=0
$$

where $n \geqq 2, n$ is odd, $a$ is continuous with $a(t) \geqq 0$, and $q(t)$ satisfies the hypothesis of Theorem 3.

In [1] G. V. Aman'eva and V. I. Balaganskii gave a sufficient condition for the nonoscillatory solutions of Equation (21) to be bounded when $q(t)=t$. This result has been extended by Lovelady [8] so that the combined results state as follows

THEOREM B. If

(i ) $\int^{\infty} t^{n-2} a(t) d t=\infty$

or (i) fails and the second order equation

$$
w^{\prime \prime}(t)+\frac{1}{(n-3) !}\left(\int_{t}^{\infty}(s-t)^{n-3} q(s) d s\right) w(t)=0
$$

is oscillatory, then every nonoscillatory solution of Equation (21) is bounded when $q(t)=t$.

Recently Lovelady [9] generalized Theorem B to Equation (21) for any $q(t) \leqq t$ provided $q^{\prime}(t) \geqq 0$ and either $q^{\prime}(t) \leqq 1$ or $q(t)-q(s) \leqq$ $t-s$. We will show that his generalization follows immediately from Theorem 3 without the upper bound restriction on $q(t)$ or its derivative. Indeed, by Theorem 3, boundedness of the nonoscillatory solutions of Equation (21) follows from the boundedness of the nonoscillatory solution of the ordinary equation 


$$
\frac{d^{n} x}{d s^{n}}+\left[a\left(Q^{-1}(s)\right) / Q^{\prime}\left(Q^{-1}(s)\right)\right] x=0 .
$$

By Theorem B, every nonoscillatory solution of Equation (22) is bounded if either

$$
\int_{0}^{\infty}\left[s^{n-2} a\left(Q^{-1}(s)\right) / Q^{\prime}\left(Q^{-1}(s)\right)\right] d s=\infty
$$

or (23) fails and the second order equation

$$
\frac{d^{2} z}{d s^{2}}+\frac{1}{(n-3) !}\left(\int_{s}^{\infty}\left[(u-s)^{n-3} a\left(Q^{-1}(u)\right) / Q^{\prime}\left(Q^{-1}(u)\right)\right] d u\right) z=0
$$

is oscillatory.

If we let $s=Q(t)$, then (23) and (24) reduce respectively to

$$
\int^{\infty} Q^{n-2}(t) a(t) d t=\infty
$$

and

$$
\begin{aligned}
& {\left[z^{\prime} / Q^{\prime}(t)\right]^{\prime}} \\
& \quad+\frac{Q^{\prime}(t)}{(n-3) !}\left(\int_{Q(t)}^{\infty}\left[(u-Q(t))^{n-3} a\left(Q^{-1}(u)\right) / Q^{\prime}\left(Q^{-1}(u)\right)\right] d u\right) z=0 .
\end{aligned}
$$

Let $v=Q^{-1}(u)$; then (26) reduces to

$$
\left[z^{\prime} / Q^{\prime}(t)\right]^{\prime}+\frac{Q^{\prime}(t)}{(n-3) !}\left(\int_{t}^{\infty}[Q(v)-Q(t)]^{n-3} a(v) d v\right) z=0 .
$$

Thus either condition (25) or the oscillation of Equation (27) when (25) fails implies that every nonoscillatory solution of Equation (21) is bounded. This is [9, Theorem 16] obtained in the form of [9, Corollary 3] without the condition $q^{\prime}(t) \leqq 1$ required in [9].

(b) Finally, we consider the second order delay equation

$$
x^{\prime \prime}(t)+a(t) x(q(t))=0
$$

where $a(t)$ is continuous with $a(t) \geqq 0$.

In [12] Wong obtained the following oscillation result

THEOREM C. If $c t \leqq q(t) \leqq t$ for some $c \in(0,1]$ and if $a(t) \geqq$ $(1+\varepsilon) /\left(4 c t^{2}\right)$, then Equation (28) is oscillatory.

We observe that for $c=1$, Theorem $\mathrm{C}$ reduces to a well-known condition for oscillation of the ordinary equation

$$
x^{\prime \prime}+a(t) x=0 \text {. }
$$


Thus, as a corresponding result for delay equations, Theorem $\mathrm{C}$ can be obtained immediately from Theorem 3. In fact, by the Corollary of Theorem 3, Equation (28) is oscillatory if the ordinary equation

$$
x^{\prime \prime}+c a(t) x=0
$$

is oscillatory. But Equation (29) is oscillatory if $c a(t) \geqq(1+\varepsilon) /\left(4 t^{2}\right)$. Hence Theorem $\mathrm{C}$ follows.

\section{REFERENCES}

1. G. V. Anan'eva and V.I. Balaganskii, Oscillation of the solutions of certain differential equations of higher order, Uspehi Mat. Nauk 14, No. 1 (1959), 135-140.

2. J. J.A.M. Brands, Oscillation theorems for second-order functional differential equations, J. Math. Anal. Appl., 63 (1978), 54-64.

3. T. A. Burton and John R. Haddock, On the delay-differential equations $x^{\prime}(t)+$ $a(t) f(x(t-r(t)))=0$ and $x^{\prime \prime}(t)+a(t) f(x(t-r(t)))=0$, J. Math. Anal. Appl., 54 (1976), 37-48.

4. I. V. Kamenev, On the oscillation of solutions of a nonlinear equation of higher order, Differencial'nye Uravnenya, 7 (1971), 927-929.

5. A. G. Kartsatos and H. Onose, A comparison theorem for functional differential equations, Bull. Aust. Math. Soc., 14 (1976), 343-347.

6. I. T. Kiguradze, Oscillation properties of solutions of certain ordinary differential equations, Dokl. Akad. Nauk SSSR, 144 (1962), 33-36 or Soviet Math. Dokl,. 3 (1962), 649-652.

7. T. Kusano and H. Onose, Oscillations of functional differential equations with retarded argument, J. Differential Equations, 15 (1974), 269-277.

8. David Lowell Lovelady, On asymptotic analysis of an odd order linear differential equation, Pacific J. Math., 57 (1975), 475-480.

9. - Oscillation and a class of linear delay differential equations, Trans. Amer. Math. Soc., 226 (1977), 345-364.

10. W.E. Mahfoud, Oscillation and asymptotic behavior of solutions of $n$th order nonlinear delay differential equations, J. Differential Equations, 24 (1977), 75-98.

11. H. Onose, A comparison theorem and the forced oscillation, Bull. Aust. Math. Soc., 13 (1975), 13-19.

12. James S. W. Wong, Second order oscillation with retarded arguments, Ordinary Differential Equations, Academic Press, New York/London, 1972, 581-596.

Received March 21, 1978 and in revised form March 20, 1979.

MurRay State University

MURRAY, KY 42071 



\section{PACIFIC JOURNAL OF MATHEMATICS}

EDITORS

DONALD BABBITT (Managing Editor)

University of California

Los Angeles, California 90024

Hugo RossI

University of Utah

Salt Lake City, UT 84112

C. C. MOORE and ANDREW OGG

University of California

Berkeley, CA 94720

\section{J. DUGUNDJI}

Department of Mathematics University of Southern California Los Angeles, California 90007

R. Finn and J. Milgram Stanford University

Stanford, California 94305

\section{ASSOCIATE EDITORS}
E. F. BECKENBACH
B. H. Neumann
F. WOLF
K. YoSHIDA

\section{SUPPORTING INSTITUTIONS}

UNIVERSITY OF BRITISH COLUMBIA

CALIFORNIA INSTITUTE OF TECHNOLOGY

UNIVERSITY OF CALIFORNIA

MONTANA STATE UNIVERSITY

UNIVERSITY OF NEVADA, RENO

NEW MEXICO STATE UNIVERSITY

OREGON STATE UNIVERSITY

UNIVERSITY OF OREGON
UNIVERSITY OF SOUTHERN CALIFORNIA

STANFORD UNIVERSITY

UNIVERSITY OF HAWAII

UNIVERSITY OF TOKYO

UNIVERSITY OF UTAH

WASHINGTON STATE UNIVERSITY

UNIVERSITY OF WASHINGTON 


\section{Pacific Journal of Mathematics}

\section{Vol. 83, No. 1 \\ March, 1979}

Richard Neal Ball, Topological lattice-ordered groups ............... 1

Stephen Berman, On the low-dimensional cohomology of some

infinite-dimensional simple Lie algebras .................. 27

R. P. Boas and Gerald Thomas Cargo, Level sets of derivatives ......... 37

James K. Deveney and John Nelson Mordeson, Splitting and modularly

perfect fields......................................

Robert Hugh Gilman and Ronald Mark Solomon, Finite groups with small

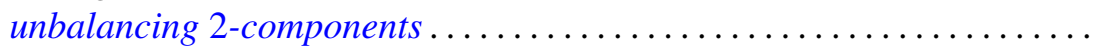

George Grätzer, Andras Hajnal and David C. Kelly, Chain conditions in free products of lattices with infinitary operations..................

Benjamin Rigler Halpern, Periodic points on tori ..................

Dean G. Hoffman and David Anthony Klarner, Sets of integers closed under

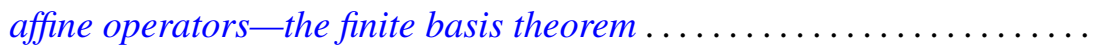

Rudolf-Eberhard Hoffmann, On the sobrification remainder ${ }^{s} X-X \ldots \ldots$

Gerald William Johnson and David Lee Skoug, Scale-invariant

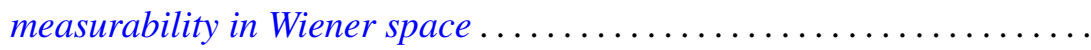

Michael Keisler, Integral representation for elements of the dual of

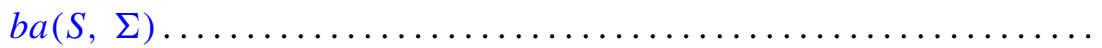

Wayne C. Bell and Michael Keisler, A characterization of the representable Lebesgue decomposition projections ................

Wadi Mahfoud, Comparison theorems for delay differential equations ...

R. Daniel Mauldin, The set of continuous nowhere differentiable functions .

Robert Wilmer Miller and Mark Lawrence Teply, The descending chain condition relative to a torsion theory...

Yoshiomi Nakagami and Colin Eric Sutherland, Takesaki's duality for regular extensions of von Neumann algebras ........ .

William Otis Nowell, Tubular neighborhoods of Hilbert cube manifolds ...

Mohan S. Putcha, Generalization of Lentin's theory of principal solutions of word equations in free semigroups to free product of copies of positive reals under addition

Amitai Regev, A primeness property for central polynomials . ...

Saburou Saitoh, The Rudin kernels on an arbitrary domain. . .

Heinrich Steinlein, Some abstract generalizations of the

Ljusternik-Schnirelmann-Borsuk covering theorem . . . 